\title{
Projeto Extensão Universitária Doutores da Beleza
}

\section{University Extension project about Beauty Doctors Proyecto de Extensión Universitaria Doctores de La Beleza}

\author{
Ana Paula Pujol ${ }^{1}$ \\ Juliana Cristina Gallas ${ }^{2}$ \\ Fabiana Thives ${ }^{3}$
}

\begin{abstract}
RESUMO
A motivação por valores ligados à responsabilidade social tem-se destacado nos últimos anos em muitas empresas preocupadas com o desenvolvimento social e político da humanidade. Através dos aspectos relacionados à caridade, compaixão $e$ amor ao próximo, inicia-se um espaço para a inclusão de valores como cidadania e participação responsável, consciente e comprometida com a comunidade, tanto dos indivíduos como das instituições. O projeto "Doutores da Beleza: inserção da beleza e estética no âmbito social" tem o objetivo de promover ações voluntárias por professores e acadêmicos do Curso Superior de Tecnologia em Cosmetologia e Estética da Universidade do Vale do Itajaí (UNIVALI) em instituições carentes: asilos, creches, orfanatos, escolas municipais e estaduais em Balneário Camboriú no Estado de Santa Catarina, desenvolvendo e ensinando atividades de corte e penteado de cabelo, unhas artísticas e maquilagens em crianças, adolescentes e idosos. Para tanto, esses trabalhos foram organizados a partir de cronograma de atividades fixas para cada instituição atendida, na qual cada uma delas apresentava necessidades distintas, sendo que o projeto possibilitou atender as demandas solicitadas. O Curso Superior de Tecnologia em Cosmetologia e Estética, através dos "Doutores da Beleza", desenvolveu desde março de 2007 ações promovendo resultados positivos tanto para o meio universitário quanto para a comunidade beneficiada, visto que o projeto se destaca na busca continuada da melhoria das condições de vida das crianças e adolescentes internadas em orfanatos e da população idosa residente em asilo.
\end{abstract}

Palavras-chave: trabalho social; voluntariado; beleza e estética.

\begin{abstract}
The motivation for values linked to social responsibility has been highlighted through the last years by companies very concerned about the political and social development of humanity. A space for the inclusion of values such as citizenship and responsible participation, aware and committed to the community, both to individuals and institutions, has been opened by means of aspects related to charity, compassion and love to the next one. The project "Doctors of Beauty: insertion of beauty and esthetics in the social field" aims at promoting voluntary action by teachers and scholars of the Graduate Course of Technology in Cosmetology and Esthetics of the University of Vale do Itajaí (UNIVALI) in deprived institutions: asylums, kindergartens, orphanages, municipal and state schools in Balneario Camboriu, in the State of Santa Catarina, developing and teaching activities of haircut and hairstyle, artistic nails, makeup in children, adolescents and elderly. Therefore, those works were organized in a fixed schedule of activities for each institution attended, and each of them had different needs. The project made possible to meet the demands requested. The Graduate
\end{abstract}

\footnotetext{
$1 \quad$ Nutricionista, professora do Curso de Cosmetologia e Estética da Univali Universidade do Vale do Itajaí - anapaulapujol@univali.br, Quinta Avenida S/N Laboratório de Cosmetologia e Estética, bloco 6A térreo - Balneário Camboriú - Santa Catarina - CEP 88330-000, fone - (47) 3261-1362/ 8844-6437.

2 Administradora; professora do Curso de Cosmetologia e Estética da Univali Universidade do Vale do Itajaí, Balneário Camboriú, Santa Catarina.jugallas@univali.br, Quinta Avenida S/N Laboratório de Cosmetologia e Estética, bloco 6A térreo - Balneário Camboriú - Santa Catarina - CEP 88330-000, fone - (47) 3261-1361/ 9929-6541.

3 Turismóloga; professora do Curso de Cosmetologia e Estética da Univali Universidade do Vale do Itajaí, Balneário Camboriú, Santa Catarina. fabimarin@redel.com.br, Quinta Avenida S/N Laboratório de Cosmetologia e Estética, bloco 6A térreo - Balneário Camboriú - Santa Catarina - CEP 88330-000, fone - (47) 8418-0839.
} 
Technology in Cosmetology and Esthetics Course, by way of the "Doctors of Beauty", has developed, since March 2007, actions promoting positive results both for the university environment and for the supported community, as the project stands out in continued pursuit of improving living conditions of children and adolescents confined in orphanages and of the elderly population residing in asylums.

Keywords: social work; volunteer; beauty and esthetics.

\section{RESUMEN}

La motivación de los valores vinculados a la responsabilidad social se ha puesto de relieve en los últimos años por empresas muy preocupado por el desarrollo político y social de la humanidad, a través de los aspectos relacionados con la caridad, la compasión y el amor al próximo, la creación de un espacio para la inclusión de motivos Por valores como la ciudadanía y la participación responsable, consciente y comprometida con la comunidad, tanto de las personas y de las instituciones. El proyecto "Los médicos de Belleza: inserción de la belleza y estética en el ámbito social" se pretende promover la acción voluntaria de los profesores y académicos de la Alta Tecnología en el Curso de Cosmetología y Estética de la Universidad de Vale do Itajaí (UNIVALI), en las instituciones privadas: Asilos, guarderías, orfanatos, escuelas, municipales y estatales en Balneario Camboriú en el Estado de Santa Catarina, y el desarrollo de las actividades de enseñanza de corte y peinado del cabello, uñas artísticas, maquilagens en niños, adolescentes y ancianos. Por estas dos obras se organizaron desde fijo calendario de actividades para cada una de las instituciones que asistieron, y cada uno de ellos tiene diferentes necesidades, y que el proyecto permitió satisfacer la demanda solicitada. El curso Superior en Tecnología Cosmética y Estética, a través de los "Médicos de Belleza" desarrollado desde marzo de 2007, la promoción de acciones de resultados positivos tanto para el medio ambiente y para beneficio de la comunidad universitaria, a medida que el proyecto se destaca en pos de mejorar las condiciones de vida de los niños Y adolescentes hospitalizados en los orfanatos y las personas de edad que residen en el asilo.

Palabras-clave: El trabajo social; voluntarios; la belleza y la estética.

Introdução

Historicamente associado a um trabalho de caráter assistencialista, paternalista e de ajuda às pessoas carentes e menos favorecidas, o trabalho social tem favorecido a autoestima, visando assim desenvolver uma concepção humanística, vinculado à responsabilidade social. Desta forma, a expressão de ética, solidariedade e participação cidadã apresenta-se como fator determinante na evolução social.

Os valores sociais relacionados à caridade e ao bem-estar social e a preocupação com a sociedade começam a ceder espaço para a inclusão e participação responsável, consciente e comprometida com a comunidade, tanto dos indivíduos como das instituições, destacando os trabalhos voluntários que são desenvolvidos por várias empresas e seus colaboradores.

Cabe ressaltar a importância de tratar esses aspectos relacionados à autoestima, de forma a introduzir uma concepção de bem-estar social aos participantes, desde os atendentes até os atendidos, o que visa viabilizar procedimentos mais abrasivos que poderão comprometer, inclusive, aspectos relacionados à saúde dos indivíduos.

O direcionamento das atividades nos ambientes pré-estabelecidos, como asilos, creches, escolas e orfanatos, remete-se ao processo conhecido como humanização a crianças, adolescentes e idosos. Esta característica de trabalho vem sendo desenvolvida, por muitos anos, pela cultura ocidental, que em larga escala prioriza a atenção aos pequenos, em detrimento daqueles que já percorreram um longo caminho.

O Projeto de Extensão Doutores da Beleza, inspirado na ONG Doutores da Alegria, é desenvolvido pelo Curso de Tecnologia em Cosmetologia e Estética, Univali, SC, e tem destaque na região do Vale do Itajaí$\mathrm{SC}$, desenvolvendo há três anos trabalhos direcionados a diversos públicos.

A diferença entre os projetos concerne nos aspectos relacionados aos objetivos indi- 
viduais. Os Doutores da Alegria têm o intuito de empregar o bom senso para ajustar a intensidade e frequência de atenção às necessidades apresentadas por quem é cuidado. Em termos pragmáticos, a observação mostra que as iniciativas de humanização hospitalar têm como foco prioritário as alas pediátricas, com a organização de verdadeiros espetáculos circenses $e$ artísticos para as crianças internadas.

Já os Doutores da Beleza têm como objetivo elevar a autoestima e bem-estar social, através de atividades relacionadas à higiene, estética e beleza. Estas, desenvolvidas em proporções que visam atender às necessidades de cada instituição visitada.

A população idosa, devido às perdas que vivencia e aos estereótipos que estão subjacentes à sua condição, podem apresentar alteração ao nível de autoestima. Essas pessoas são propensas a subestimar ou sobrestimar as suas competências, sendo que essa tendência tem sido interpretada como um mecanismo protetor. $\mathrm{O}$ ajuste com o sucesso, na terceira idade, depende frequentemente da capacidade do idoso para manter padrões pessoais que sejam compatíveis com as normas sociais. As atividades como corte de cabelos, manicure, pedicure e maquilagem desenvolvidas pelos Doutores da Beleza podem contribuir para a melhora da autoestima e manutenção do padrão de beleza social, melhorando sua qualidade de vida.

Em orfanatos, percebe-se a necessidade de trabalhar atividades relacionadas ao lúdico, com decoração de unha, maquilagem artística, teatro, brincadeiras de roda, desfiles de moda, destacandoainda a importância da dinamicidade, potencializando a criatividade de cada criança e adolescente da instituição.

Nas escolas, o projeto foi diferenciado, ensinando-se aos adolescentes a prática que envolve os serviços de manicure, corte $e$ modelagem de cabelo, maquilagem, através de oficinas práticas e palestras, com orientações de possibilidades de mercado trabalho.
$\mathrm{Na}$ tradição do trabalho voluntário, o hospital sempre foi um espaço desse tipo de iniciativa. Ainda se fazem bastante presentes ações com forte caráter religioso e assistencialista. Trabalhos mais sistemáticos, com capacitação dos voluntários e com caráter de responsabilidade social, estão em fase bastante inicial. No entanto, mesmo com essa tradição, o número de iniciativas é pequeno frente às necessidades das instituições.

Os aspectos que visam diferenciar o projeto de Extensão Doutores da Beleza caracterizam-se na formulação de atividades a partir das necessidades de cada ambiente trabalhado. Exatamente pelos motivos expostos é que os conceitos e práticas de humanização envolvendo atividades sociais precisam ser atualizados.

Mais do que um atendimento de assistencialismo, o projeto visa desenvolver a integração dos envolvidos - como alunos, professores, colaboradores e comunidade atendida -, além dos aspectos que envolvem as ações práticas.

Tem-se também a importante colaboração dos conhecimentos acadêmicos, com preparo para desenvolver atividades relacionadas ao bem-estar social, assim aprimorando o comprometimento voluntário, ética e cidadania que envolvem este tipo de ação.

Por se tratar de aspectos que envolvem a autoestima, é importante destacar a concepção de Ladd et al. (1996), que fazem uma observação relacionada com a melhora da autoestima $e$ imagem pessoal, aspecto este que pode favorecer a empatia, destacando que quanto mais empática é uma criança, mais competente socialmente ela é capaz de ser. Esse achado corrobora estudos anteriores, que revelam que crianças socialmente competentes tendem a ser sensiveis e empáticas com seus pares, capazes de estabelecer relações de amizade e habilidosas na resolução de problemas (HOWES et al., 1994). Uma vez que a empatia favorece o vínculo entre 
as pessoas, espera-se que ela contribua para o desenvolvimento das relações de amizade, que, em situações de stress, funcionam como apoio social e auxiliam na capacidade de adaptação (LADD et al., 1996).

A atuação da universidade junto à comunidade de Balneário Camboriú e região se justifica na medida em que a instituição detém conhecimento e recursos humanos capacitados para desenvolver atividades que possam contribuir para uma melhor qualidade de vida e exercício da cidadania.

Sendo a Universidade também uma instituição educacional, é premente a necessidade de compatibilizar as atividades de ensino com as atividades de voluntariado, no sentido de preparar aqueles que passam pelos seus bancos para o exercício profissional como uma ação digna de compromisso social com o outro.

O projeto Doutores da Beleza vem buscando nortear-se cada vez mais pelos pressupostos da interdisciplinaridade, que, segundo Fazenda (1991), referem-se ao encontro participativo de todos os segmentos que compõem essa construção, tendo como relevante a democratização das relações sociais, onde os sujeitos devem estar permanentemente mobilizados para discussões, encaminhamentos e resolução de problemas.

Para tanto, compreendemos que a interdisciplinaridade envolve os princípios da indissociabilidade entre extensão, ensino $e$ pesquisa, bem como a participação das diferentes áreas do conhecimento, pois esta troca enriquece as ações práticas e reflexivas.

Ressalta-se também que as práticas interdisciplinares favorecem a formação acadêmica, possibilitando uma visão prospectiva de trabalho coletivo, assim como relaciona a produção intelectual às ações de extensão. Neste sentido, um projeto integrado requer a construção de grupos de estudos que apresentem e discutam a literatura da área, oportunizando assim a produção de textos científicos, os quais possam ser referência para as atividades acadêmicas do ensino, posto que a participação de acadêmicos em projetos dessa natureza fornece à sua formação um caráter investigativo.

É nesta perspectiva de trabalho integrado, estabelecendo "pontes" entre diversas áreas de conhecimento, que esse projeto de extensão se coloca, configurando-se, então, enquanto desafio interdisciplinar. Essa proposta visa melhorar a qualidade da assistência à saúde e educação prestada ao indivíduo, família e comunidade, bem como colaborar para a preservação do meio ambiente e das condições de moradia dos indivíduos.

O Curso Superior de Tecnologia em Cosmetologia e Estética, inserido no Projeto de Extensão Doutores da Beleza, pôde desenvolver ações que promoveram resultados positivos tanto para o meio universitário quanto para a comunidade beneficiada, visto que o projeto em vigência compartilhou interesses em comum quando se destaca a busca continuada da melhoria das condições de vida das crianças hospitalizadas, internadas em orfanatos e população idosa residente em asilo.

\section{Relato de experiência}

As atividades dos Doutores da Beleza iniciaram em 2007 e permanecem nos dias atuais. Semestralmente, a proposta é apresentada a instituições como orfanatos, asilos, escolas públicas e hospitais. No aceite, um termo de ciência é assinado pelo responsável da instituição e um cronograma de atividades e datas é elaborado, conforme a necessidade e disponibilidade da instituição.

O projeto tem apoio de várias empresas do ramo da Cosmética e Beleza, ligadas ao Curso Superior de Tecnologia em Cosmetologia e Estética, na doação de uniformes, maquilagens, esmaltes, tesoura para corte de cabelos e outros materiais necessários. 
Enfatizando a interdisciplinariedade, cursos de graduação como Moda e Design, da mesma instituição, colaboram na criação $e$ confecção dos uniformes (foto 1) e elaboração da logomarca (figura 1), respectivamente.

As atividades são desenvolvidas duas vezes por semana, de acordo com o cronograma inicialmente firmado. Quatro a seis acadêmicos participam voluntariamente, sempre acompanhados de um professor responsável. Essas atividades duram de duas a quatro horas e são desenvolvidas no período matutino $e$ vespertino. Os trabalhos realizados são: corte de cabelo, penteado, escova, maquilagem, serviços de manicure, pedicure e maquilagem artística, sempre considerando aspectos de higiene, biossegurança e ética. Em anexo, a foto 3 mostra atividades desenvolvidas na Escola Estadual Francisca Gevaer; as fotos 4 e 5, no Asilo Lar dos Idosos e as fotos 6 e 7 no Orfanato Casa da Criança, respectivamente. Até o momento, 650 atendimentos foram realizados, contando com a participação de professores e alunos voluntários, bem como bolsistas do projeto.

Em relação à divulgação do projeto, ela é feita a partir de estratégias como participação em programas de televisão, rádio, reportagens em jornais e revistas locais e regionais. Essa divulgação favorece o incentivo nas doações para os locais atendidos e para o recurso financeiro para o desenvolvimento das atividades.

\section{REFERÊNCIAS}

FAZENDA, I. Práticas interdisciplinares na escola. São Paulo: Cortez, 1991.

HOWES, C.; MATHESON, C. C.; HAMILTON, C. E. Maternal, teacher, and child care history correlates of children's relationships with peers. Child Development, v. 65, p. 264-273, 1994.

LADD G. W.; KOCHEDENFER, B. J.; COLEMAN, C. C. Friendship quality as a predictor of young children's early school adjustment. Child Development, v. 67, p. 1103-1118, 1996.

\section{Considerações finais}

O projeto Doutores da Beleza almeja a participação dos professores e acadêmicos, assim como das instituições ligadas a ele, e obtém resposta com êxito, o que proporcionou credibilidade nas atividades desenvolvidas. Através da inserção da prática da cosmetologia e estética no âmbito social, o Curso Superior de Tecnologia em Cosmetologia e Estética da Univali pode atender às expectativas da Instituição, do curso e da comunidade nos seguintes aspectos:

- Melhora da autoestima dos idosos e crianças do orfanato;

- Humanização e integração dos alunos das escolas com a estética e beleza;

- Crescimento profissional e pessoal pelos dicentes;

- Desenvolvimento de atividades de extensão universitária pelos discentes.

Constata-se a importância de que mais instituições e empresas desenvolvam projetos similares, com objetivos direcionados à melhora do bem- estar social e à autoestima de entidades e comunidades carentes.

NUNES, E. D. Interdisciplinaridade: conjugar saberes. Saúde em Debate, v. 26, n. 62, p. 249-258, set/dez. 2002.

RECADASTRAMENTO ANO 2005. Focos de Ocupação Irregular Município de Balneário Camboriú - Caderno 07. Secretaria da Mulher, Criança, Adolescente, Idoso, Trabalho e Desenvolvimento Comunitário. Balneário Camboriú, 2005.

Texto recebido em 21 de janeiro de 2009. Texto aprovado em 26 de março de 2009. 


\section{Anexo}

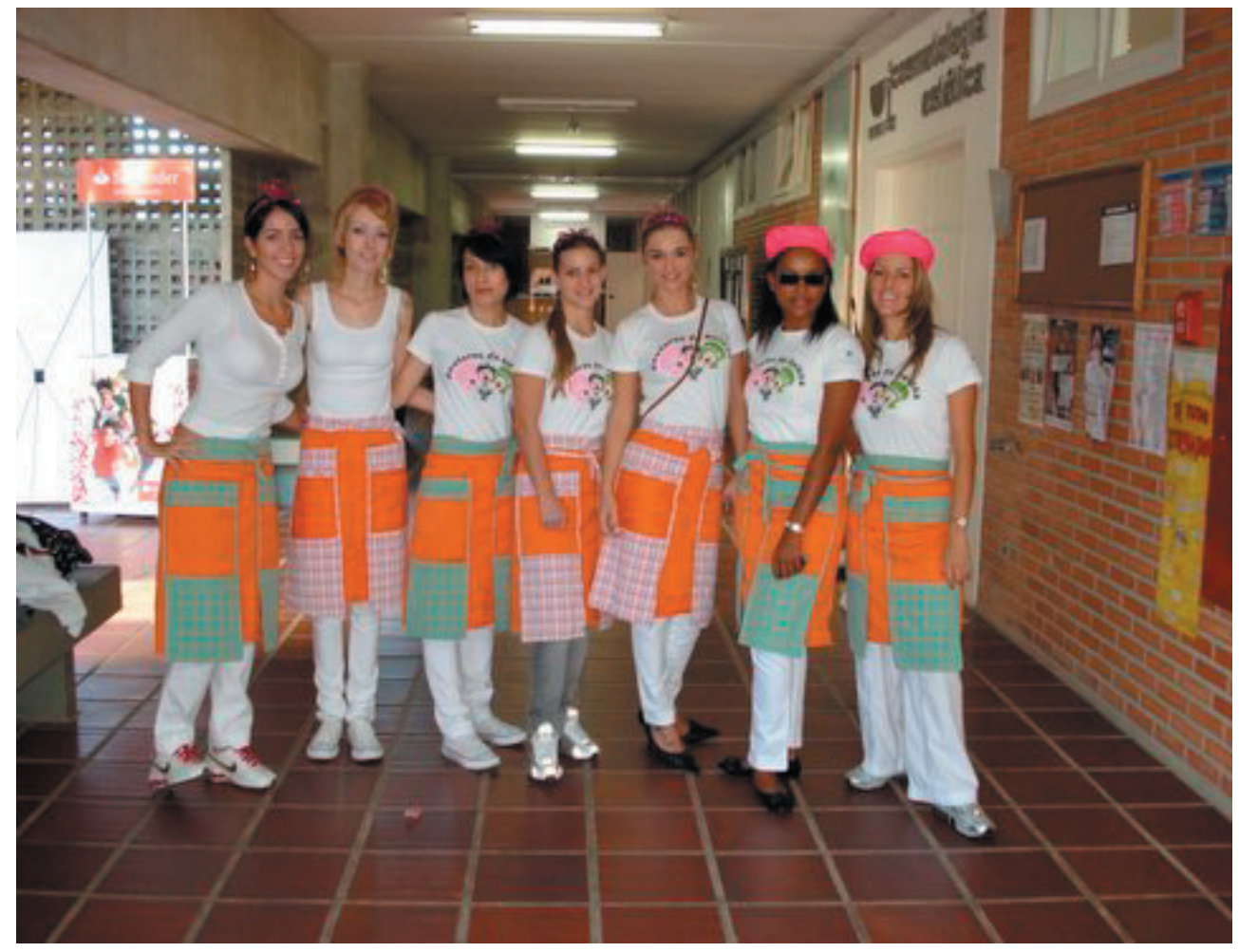

FOTO 1 - ACADÊMICOS DE COSMETOLOGIA E ESTÉTICA COM O UNIFORME DOS DOUTORES DA BELEZA ELABORADOS PELO CURSO DE MODA DA UNIVALI, 2009.

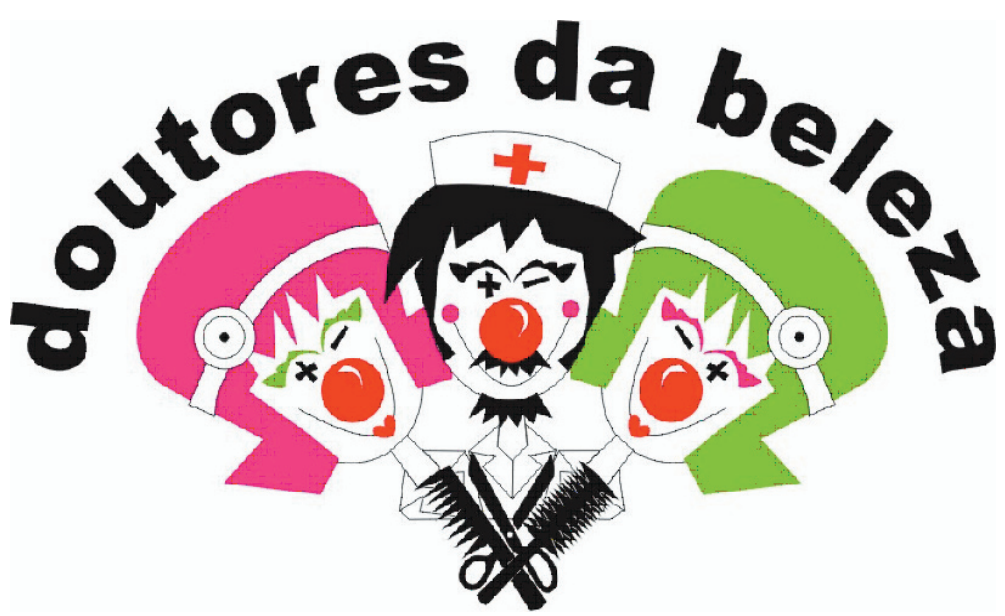

FIGURA 1 - LOGOMARCA DO PROJETO DE EXTENSÃO DOUTORES DA BELEZA, ELABORADA PELOS ACADÊMICOS DO CURSO DE DESIGN DA UNIVALI, 2007. 